\title{
Contraintes Organisationnelles Et Resilience Chez Le Fonctionnaire Du Ministere Ivoirien De La Fonction Publique
}

\author{
Gacha Franck-Gautier \\ Université Peleforo Gon Coulibaly de Korhogo (Côte d’Ivoire) \\ Assouman Ahou Mélissa Estelle \\ Université Félix Houphouët-Boigny de Cocody (Abidjan)
}

doi: 10.19044/esj.2017.v13n11p237 URL:http://dx.doi.org/10.19044/esj.2017.v13n11p237

\begin{abstract}
Ivorian public Service is subject to criticism. To overcome this, reforms are undertaken to enhance its level of service. However, they apply at the expense of the human factor, which is essential to motivate. This communication builds on previous work, supplemented by semi-structured interviews with Ivorian officials. A content analysis of the discourse of actors was performed. It appears that the delay, corruption and absenteeism resulting from low wages as a source of motivation, as well as the lack of transparency on the decisions of the administrative organization and the small delegation of responsibilities. In a word, the administrative staff is always underway in the Public Service Ivorian. However, the human factor needs to trade, listening, evaluation of performance, information, motivation, mobilization.
\end{abstract}

Keywords: Public Service, human Factor, motivation, absenteeism, resilience.

\section{Résumé}

La Fonction publique ivoirienne est l'objet de critiques. Pour pallier ce fait, des réformes sont entreprises pour rehausser son niveau de prestations. Mais, celles-ci s’appliquent au détriment du facteur humain, ressource essentielle à motiver. Le présent article s'appuie sur des travaux antérieurs, complétés par des entretiens semi-structurés avec des fonctionnaires ivoiriens. Une analyse du contenu du discours des acteurs a été réalisée. Il ressort que le retard, la corruption et l'absentéisme résultent du faible niveau de salaire qui constitue une source de démotivation, de même que l'insuffisance de transparence sur les décisions de l’organisation administrative et la faible délégation des responsabilités. En un mot, la 
gestion administrative du personnel a toujours cours dans la Fonction publique ivoirienne. Or, le facteur humain a besoin d'échanges, d'écoute, d'évaluation de sa performance, d'information, de motivation, de mobilisation.

Mots-clés : Fonction publique, facteur humain, motivation, absentéisme, résilience.

\section{Introduction}

La Fonction publique est généralement l’objet de critiques diverses : faible rendement, absentéisme, retard des agents, dépenses excessives, lourdeur (Gacha, 2010). Ces critiques sont fondées si l'on se réfère à la multiplicité de structures et de services interdépendants, mais aussi à la complexité de l'itinéraire administratif emprunté par les requêtes des usagers. C'est pour pallier ces irrégularités que la Côte d’Ivoire va initier des opérations de réforme administrative et externaliser des activités auparavant dévolues à des fonctionnaires. Ces actions de réforme auront pour objectif de rehausser le niveau des prestations et de performance de la Fonction publique (Gacha, 2012).Cet objectif ne saurait être atteint qu'à travers l'adoption de nouveaux outils d'évaluation, de motivation et de promotion de l'élément humain qui constitue une ressource incontournable. C'est à ce niveau que se pose la problématique de la gestion des ressources humaines dans l'administration publique, en termes de gestion des compétences, des carrières, des emplois et des effectifs.

En effet, à l'instar du secteur privé, la politique GRH devient un enjeu déterminant de la performance du secteur public (Benabbou, 2001).La réforme administrative est réalisée par et avec les fonctionnaires en faveur des usagers et autres prestataires. Aussi, est-il important que le fonctionnaire soit convaincu de l'opportunité de la réforme administrative et que ses besoins soient pris en considération lors de la réforme (MFPRA, 2004). Cette assertion est d'autant plus vraie que l'on a commencé à intégrer la dimension humaine dans les réformes administratives quand ont surgi des comportements de corruption, de pots-de-vin, d’absentéisme, ou toute autre forme de comportements de compensation. Ces adaptations comportementales exigent une restructuration en profondeur du service public et une lecture plus attentive de la situation des fonctionnaires, loin des préjugés.

Les réformes implémentées actuellement dans l’organisation administrative ivoirienne mettent en avant la moralisation des agents, la transparence et la bonne gouvernance. Mais, là encore, se pose le problème des priorités: le constat est que les priorités dégagées par l’appareil administratif ne coïncident pas avec celles des fonctionnaires. Les acteurs en 
présence définissent chacun des actions qui, prises en compte et appliquées, pourraient favoriser un meilleur fonctionnement du système administratif. Ainsi, pendant que l’organisation définit des priorités (moralisation de la vie publique, transparence, lutte contre la corruption,...), les fonctionnaires en font de même (amélioration des conditions de travail, augmentation salariale,...). Une étude du Ministère de la Réforme Administrative (2005) révèle aussi que les fonctionnaires ne pourront réellement s’impliquer dans le processus de changement que si le système de rémunération devient plus attrayant, plus motivant.

Ainsi, la préoccupation centrale demeure-t-elle de questionner les facteurs organisationnels qui favorisent la résilience chez les fonctionnaires ivoiriens. En d'autres termes, comment les fonctionnaires s'adaptent-ils aux contraintes organisationnelles au point où tous semblent accepter les irrégularités constatées ? Les comportements adoptés sont-ils le résultat de la méconnaissance des textes réglementaires ou plutôt une réponse légitime devant les revendications corporatives non satisfaites? Tels sont les questionnements de cet article qui se propose d'identifier les contraintes organisationnelles qui (re)modèlent les comportements des fonctionnaires ivoiriens. De façon spécifique, l'article entend fournir des informations sur les facteurs de la résilience chez les agents publics de façon générale et ceux du Ministère de la Fonction publique en particulier. L’article est structuré autour d'une méthodologie, de résultats et analyses suivis d'une discussion. La conclusion revient sur les résultats obtenus tout en donnant un éclairage sur les portées sociale et scientifique de l’étude.

\section{MATERIEL ET METHODES}

Le présent article se propose d'identifier les contraintes organisationnelles qui influencent les comportements des fonctionnaires ivoiriens. A cet effet, l'hypothèse suivante est élaborée : les comportements des travailleurs sont liés aux attitudes vis-à-vis des textes réglementaires, mais également au rapport au salaire et aux sollicitations sociales. Cette double perspective empirique exige une double analyse pour mieux ressortir les comportements des acteurs, les sens et significations qu'ils donnent à leurs actions.

\section{Matériel}

Le matériel utilisé reste pour l'essentiel l'entretien semi-structuré et l'observation des fonctionnaires sur le lieu de travail. L'article se fonde aussi sur des travaux de recherche que nous avons réalisés antérieurement et auxquels nous avons adjoint des données issues d'une nouvelle documentation composée d'articles scientifiques et du Statut général de la Fonction publique qui définit les conditions de la vie administrative. Cette 
documentation a été complétée par cinq (5) entretiens semi-structurés que nous avons eus avec des responsables de la Fonction publique ivoirienne. Les thèmes abordés ont été essentiellement la question de la gestion des ressources humaines, la situation statutaire et la résilience des travailleurs ainsi que les leviers à actionner pour les motiver.

\section{Méthodes}

Pour recueillir les informations auprès des responsables administratifs, des entretiens individuels libres ont été réalisés. La méthode utilisée se résume à la collecte et à l'analyse des données qualitatives. De façon concrète, nous avons procédé à la transcription intégrale des entretiens réalisés puis à une catégorisation des idées. A partir de cette catégorisation, une analyse du contenu du discours des acteurs a été réalisée afin de ressortir des éléments explicatifs de la résilience des fonctionnaires ivoiriens. Les enquêtes de terrain se sont déroulées du 17 au 24 août 2016 au siège du Ministère de la Fonction Publique et de la Modernisation Administrative (MFPMA), dans la commune du Plateau, à Abidjan.

\section{RESULTATS ET ANALYSES}

\section{Les facteurs de démotivation des fonctionnaires ivoiriens}

Il ressort des entretiens avec les responsables du MFPMA que le forum social organisé en 2007 à Grand-Bassam (ville de la basse-côte ivoirienne) a fait des recommandations dont l'application a commencé depuis fin 2008. Les propos d'un Chargé d’Etudes à la Direction Générale de la Fonction Publique nous situent mieux sur la question :

"le forum social a préconisé une réforme salariale et indemnitaire et c'est ce qu'on a commencé avec les enseignants du secondaire et du supérieur, avec les médecins, avec les infirmiers, avec les agents des eaux et forêts, les agents des douanes, les agents des affaires maritimes, et cela devait toucher l'ensemble des fonctionnaires. Seulement, on n'a pas pu l'étendre à tous les fonctionnaires compte tenu des contraintes liées au processus de sortie de crise qui englobent une forte partie du budget de l'État et qui nous empêchent ou qui ne nous permettent pas d'exécuter toutes les réformes salariales et indemnitaires $»$.

En mai 2015, à l'occasion de la Fête du travail, le Chef de l'Etat a annoncé officiellement le déblocage des avancements pour tous les fonctionnaires avec effets immédiats. Ce déblocage a été diversement 
apprécié par les fonctionnaires: pour certains, le salaire a connu une amélioration significative et pour d'autres, il y a eu des ponctions réductrices du salaire antérieur.

Outre le niveau de traitement salarial actuel qui constitue une source de démotivation, nous pouvons mentionner les frustrations subies par les agents de la part de leurs supérieurs hiérarchiques. Les fonctionnaires interrogés dénoncent une communication insuffisante sur les décisions de leur organisation, une faible délégation des responsabilités, mais aussi l'arbitraire dans les prises de décisions qui les concernent. Si l'on se réfère aux dires d'un agent administratif interrogé au MFPMA, on se rend compte qu'il existe un réel déficit d'informations quant à l'élaboration des politiques de réformes : "Nous, on n'est jamais au courant de rien; pourtant on a un syndicat qui est censé nous tenir informés de tout ce qui se passe; on ne nous informe pas et puis quand ils arrêtent leurs décisions de réformes, on nous demande de les appliquer. Nous ne sommes pas des robots; le syndicat même ne sait pas quand les réformes sont élaborées ». Concernant la faible délégation des responsabilités aux travailleurs, un sous-directeur au MFPMA confirme cet état de fait : " «la Fonction publique a un effectif important de travailleurs; pour rapprocher l'autorité des agents, on a cru bon de conférer des pouvoirs à un nombre élevé d'intellectuels afin d'organiser et suivre le travail qui se fait au bas de l'échelle ».

Concernant le volet de la formation, les enquêtés sont unanimes pour dire que des sessions sont organisées, des séminaires et autres colloques de mise à niveau. Ces formations sont généralement organisées pour les cadres supérieurs (grades A4 à A7) et les cadres moyens (grades A1 à A3). Il existe à cet effet une Direction de la Formation et des Stages au sein du Ministère de la Fonction Publique et de la Modernisation Administrative qui étudie et planifie les thèmes à traiter. Il arrive aussi que chaque département ministériel ou un service public quelconque, en fonction de ses besoins et de ses moyens, organise des sessions de formation à l'endroit de ses travailleurs. En guise d'exemple, nous avons, courant janvier 2011, participé à une formation à l'intention des fonctionnaires de l'Office National d’Identification $^{45}$ sur la gestion des carrières. La formation a consisté à clarifier le concept de carrière ainsi que les enjeux de la gestion des carrières des agents de cette structure publique. Il y a également la formation professionnelle qui est initiée pour les fonctionnaires reçus à un concours professionnel interne.

Contrairement aux entreprises privées, la Fonction publique accorde peu de promotions aux travailleurs. Cela trouve sa justification dans l'incidence budgétaire que les promotions impliquent. De façon explicite, il

\footnotetext{
${ }^{45} \mathrm{ONI}$, structure rattachée au Ministère d’Etat, Ministère de l’Intérieur et de la Sécurité.
} 
faut savoir que toute promotion d'un agent s'accompagne nécessairement d'une retombée financière pour ce dernier. Or, l'administration publique s'efforce de maîtriser son budget de fonctionnement en évitant autant que possible d'y greffer des charges supplémentaires. Ceci expliquant cela, la Fonction publique ne promeut pas souvent ses travailleurs. Toutefois, l'organisation accorde des promotions, mais dans des proportions très limitées.

Par ailleurs, la Fonction publique, avant le forum social de 2007, n’accordait ni gratifications ni primes. Les régies financières structures qui octroyaient des primes à leurs agents depuis 2001) le faisaient parce qu'elles génèrent par elles-mêmes des revenus. Toutefois, à l'issue du forum social, des propositions ont été faites allant dans le sens de la généralisation des primes à l'ensemble des fonctionnaires et agents de l'État de Côte d'Ivoire. Depuis 2008, des primes trimestrielles sont donc versées aux fonctionnaires, vu qu'ils génèrent des revenus grâce aux actes administratifs payants délivrés aux usagers. Les fonds ainsi générés sont gérés par un agent comptable du Trésor public détaché dans le service en question. Paradoxalement, les textes de la Fonction publique et ceux des finances publiques n'autorisent pas le versement de primes aux fonctionnaires ; ces mesures exceptionnelles sont prises pour motiver les travailleurs.

Soulignons aussi que les avancements dans l'organisation administrative se font tous les deux ans en fonction de la notation des agents. Pour illustration, si un agent est bien noté, par exemple 4 sur 5, le délai pour qu'il bénéficie de l'avancement est moins long que celui des autres fonctionnaires qui ont une note inférieure à 3. C'est en quelque sorte une forme de bonification que la Fonction publique applique.

\section{La spécificité de la Gestion des Ressources Humaines dans la Fonction publique ivoirienne}

La Fonction publique accomplit ses missions en s’appuyant sur la communauté d'hommes et de femmes qui en constituent le socle. C'est dire que l'Homme est incontournable à maints égards. Comment les Ressources Humaines sont-elles gérées au niveau de la Fonction publique ivoirienne ? Commençons par apprécier les propos d'un sous-directeur de la Direction des Opérations de Réformes, de l'Evaluation et de la Banque des Données (DOREBD) :

«De façon générale, pour l'épanouissement du fonctionnaire, au niveau de la Fonction publique, bon... moi, je peux te dire qu'il n'y a pas grandchose (...). Dire qu'il y a un programme social de construction de logements pour les fonctionnaires, ou bien d'amélioration des conditions de vie, 
véritablement, en ma connaissance, il n'y a pas

grand-chose ».

La Fonction publique ivoirienne est dotée de services de gestion des ressources humaines qui ont des dénominations différentes en fonction de l'effectif des ministères. Ainsi, les grands ministères disposent d'une Direction des Affaires Administratives et Financières (DAAF) et d'une Direction des ressources humaines (DRH), les moins grandes seulement d'une DRH et les plus petites d'une Direction du personnel. En fait, ces appellations n’ont aucune incidence véritable sur la gestion des ressources humaines puisque ces structures font pratiquement la même chose, occultant la prospective. Les directeurs de ces structures se contentent de constituer les dossiers pour chaque agent, d'établir les notes de service, les notes pour le départ en congé, etc. En un mot, c’est la gestion classique des ressources humaines (la gestion administrative du personnel) qui est pratiquée dans l'administration publique ivoirienne. Par conséquent, c'est le modèle objectivant de la gestion des ressources humaines qui est en vigueur dans la Fonction publique ivoirienne, étant donné qu'elle a une configuration mécaniste, au sens de Mintzberg (1986). Or dans le quotidien, le facteur humain est permanent et il prend le nom d'échange, d'écoute, d'expression, d'évaluation de la performance, d'information, d'explication, de motivation, de mobilisation. Ce que le gestionnaire des ressources humaines doit être à même d'accomplir de façon permanente.

Le fonctionnaire est dans une situation statutaire vis-à-vis de l'administration publique. Lors du recrutement, généralement par voie de concours, les postes et les responsabilités ne sont pas négociables: le fonctionnaire n’a pas de volonté, il peut servir partout, être affecté partout ou maintenu en un lieu, même en temps de guerre. "C'est le sacerdoce, on ne demande pas ton avis » a dit le sous-directeur de la DOREBD.

La nomination à des postes de responsabilité relève de la « compétence discrétionnaire »de l'autorité qui a pouvoir de nommer, sans demander l'avis de l'intéressé. Il n’y a pas de critères objectifs pour l'affectation d'un agent à un poste ; cela est laissé à l'appréciation du chef de service qui décide d'affecter un agent dans tel ou tel service ou lui confier telle ou telle responsabilité. Toutefois, pour les postes de responsabilité, il y a des critères. A titre d'exemple, pour être sous-directeur dans la Fonction publique, il faut être de la catégorie A, grade minimum A3.

Pour ce qui est des prestations dont bénéficie le fonctionnaire, il n'existe pas d'autres couvertures en dehors de l'assistance médicale fournie par la Mutuelle Générale des Fonctionnaires et Agents de l'État de Côte d'Ivoire (MUGEF-CI). Cette assistance consiste en la délivrance de bons de produits pharmaceutiques aux fonctionnaires malades ou à leurs ayantsdroits. Ce qui leur permet de se procurer les médicaments à hauteur de 30\% 
du montant, la mutuelle endossant $70 \%$ du montant. Là encore, il y a des restrictions qui sont faites dans les prestations : certaines pharmacies refusent les bons de produits pharmaceutiques; le fonctionnaire malade est donc obligé de sillonner plusieurs pharmacies pour en trouver une qui accepte de servir les bons MUGEF-CI. Par ailleurs, certains médicaments (antibiotiques, sérums intraveineux, etc.) n'étant pas supportés par les bons de produits pharmaceutiques, le fonctionnaire est tenu de s'en procurer à ses propres frais. Enfin, sur toute l'étendue du territoire ivoirien, il existe un seul service médical dédié aux agents publics, avec plusieurs unités médicales pour la prise en charge du travailleur de l'Etat. Pour pallier ces insuffisances, certains services publics mettent en place des structures destinées à assurer des prestations de qualité à leurs membres. C'est le cas des amicales et mutuelles qui ont été créées au sein du Trésor public, de la Direction Générale des Impôts, de la Police Nationale, des Forces armées, des Douanes, de la Direction Générale du Travail.

\section{CONCLUSION}

Il faut dire que les textes de l'administration publique, matérialisés par le Statut général de la Fonction publique, définissent au préalable les rapports de l'organisation à l'égard des travailleurs. Ces derniers, dans une situation dite statutaire vis-à-vis de la Fonction publique, ne peuvent qu'être conditionnés par les textes en vigueur: le poids des structures est plus qu'évident. Cette réalité est d'autant plus avérée que les textes influencent également le mode de gestion des ressources humaines de la Fonction publique. Ce sont ces mêmes textes qui consacrent la résilience des fonctionnaires ivoiriens. En même temps qu'ils limitent les actions et initiatives des fonctionnaires, ces textes constituent le catalyseur des comportements de compensation que l'on observe chez les différents agents publics, notamment la corruption, les pots-de-vin, l'absentéisme, la désertion rapide des bureaux, les activités lucratives parallèles. Ces adaptations comportementales exigent une restructuration en profondeur du service public et une lecture plus attentive de la situation des fonctionnaires, loin des préjugés, parce qu'ils sont en amont et en aval de toute la procédure organisationnelle d'un Etat.

\section{References:}

1. Benabbou, A. (2001). «La Gestion des Ressources Humaines : une nouvelle vision au service d'une Administration moderne ", in Aourach : bulletin d'information du Secrétariat d'État à l'Habitat, $\mathrm{n}^{\circ} 9$, Rabat. [En ligne]. Consulté le 12 juillet 2016. Disponible sur http://www.seh.gov.ma 
2. Gacha, F. G. (2010). Motivation et assiduité au travail chez des fonctionnaires du Trésor public. Mémoire de fin de cycle en psychologie appliquée non publié, CIERPA, Université de Cocody, Abidjan.

3. Gacha, F. G. (2012). Réformes administratives et quête de performance dans le secteur public ivoirien : cas du Ministère de la Fonction Publique et de la Réforme Administrative. Thèse de doctorat unique Sociologie non publiée, Université Félix HouphouëtBoigny de Cocody, Abidjan.

4. MFPRA, (2004). "Bilan de la réforme administrative pour la période allant de septembre 1999 à décembre 2003 », Luxembourg. [En ligne]. Consulté le 9 juillet 2016. Disponible sur http://www.mfpra.public.lu

5. Ministère de la Réforme Administrative, (2005). Élaboration d'un code d'éthique et de déontologie commun à tous les agents de l'État de Côte d'Ivoire. Rapport final. Abidjan. MINRAD.

6. Mintzberg, H. (1986). Le pouvoir dans les organisations (traduit par Paul Sager). Paris. Les Éditions d'Organisation et Les Éditions Agence d'Arc. 